\title{
On the breakup of an air bubble injected into a fully developed turbulent flow. Part 2. Size PDF of the resulting daughter bubbles $\dagger$
}

\author{
By C. MARTÍNEZ-BAZÁN, \\ J. L. MONTAN̈ÉS AND J. C. LASHERAS \\ Department of Mechanical and Aerospace Engineering, \\ University of California, San Diego, La Jolla, CA 92093-0411, USA
}

\begin{abstract}
Based on energy principles, we propose a statistical model to describe the bubble size probability density function of the daughter bubbles resulting from the shattering of a mother bubble of size $D_{0}$ immersed in a fully developed turbulent water flow. The model shows that the bubble size p.d.f. depends not only on $D_{0}$, but also on the value of the dissipation rate of turbulent kinetic energy of the underlying turbulence of the water, $\epsilon$. The phenomenological model is simple, yet it predicts detailed experimental measurements of the transient bubble size p.d.f.s performed over a range of bubble sizes and dissipation rates $\epsilon$ in a very consistent manner. The agreement between the model and the experiments is particularly good for low and moderate bubble turbulent Weber numbers, $W e_{t}=\rho \Delta u^{2}\left(D_{0}\right) D_{0} / \sigma$ where the assumption of the binary breakup is shown to be consistent with the experimental observations. At larger values of $W e_{t}$, it was found that the most probable number of daughter bubbles increases and the assumption of tertiary breakup is shown to lead to a better fit of the experimental measurements.
\end{abstract}

\section{Introduction}

The dispersion of an immiscible fluid in a turbulent one is commonly found in many engineering as well as natural processes. The size distribution of the drops for bubbles) resulting from the turbulent breakup and the dynamics of their interactions with the underlying turbulence plays a determinant role in the overall performance of these processes. For example, in liquid-liquid or gas-liquid chemical separators, the absorption rate of a given chemical compound depends not only on the dynamics of the motion between the two immiscible fluids, but more importantly on the size p.d.f. of the drops (or bubbles) in which one fluid is dispersed in the other. Surface-dominated diffusion processes of this nature are also found extensively in naturally occurring phenomena, i.e. air bubbles entrained by the air-sea interaction contribute to the exchange of gases and water vapour between the oceans and the atmosphere (Thorpe 1982; Farmer \& Vagle 1988; Longuet-Higgins 1992; Melville 1996). A quantitative understanding of drop (or bubble) breakup and coalescence is essential to the development of predictive models for the behaviour of these processes.

Resulting from its widespread use, the problem of the breakup of an immiscible fluid immersed in a turbulent flow has been the subject of a continuing investigation 
and has generated a large bibliography (Kolmogorov 1949; Hinze 1955; Coulaloglou \& Tavlarides 1977; Konno, Aoki \& Saito 1983; Cohen 1991; Longuet-Higgins 1992; Tsouoris \& Tavlarides 1994; Novikov \& Dommermuth 1997, among others).

The framework of the 'population balance equation', given as equation (3.1) of a companion paper (Martínez-Bazán, Montañés \& Lasheras 1999, referred to hereafter as Part 1), has been widely used in chemical engineering, combustion (spray equation), cloud dynamics etc. to study two-phase processes dominated by breakup and coalescence (Coulaloglou \& Tavlarides 1977; Williams 1985; Prince \& Blanch 1990; Tsonoris \& Tavlarides 1994; Sathyagal \& Ramkrishna 1996; etc). This Boltzmanntype equation describes the time rate of change of the number density of drops (or bubbles) with respect to position and time of a certain size $D$ existing in a time $t$, at a given position $\boldsymbol{x}, n(D, \boldsymbol{x}, t)$, (Williams 1985):

$$
\frac{\partial n}{\partial t}+\nabla_{x} \cdot(v n)=-\frac{\partial}{\partial D}(R n)+\dot{Q}_{b}+\dot{Q}_{c},
$$

where $v$ is the mean velocity of all the particles of size $D, R=\mathrm{d} D / \mathrm{d} t$ is the rate of change of the size $D$ of a particle due condensation, evaporation and dissolution, $\dot{Q}_{b}$ and $\dot{Q}_{c}$ are the rate of change of $n$ due to the breakup and coalescence respectively. For very dilute systems (negligibly small rate of collisions between particles), and in the absence of dissolution (or evaporation) effects, the rate of change of the number density of particles can simply be written as the sum of the death rate of particles of size $D$ due to their breakup into smaller ones, and the birth rate of particles of size $D$ resulting from the breakup of larger ones:

$$
\frac{\partial n}{\partial t}+\nabla_{x} \cdot(v n)=\int_{D}^{\infty} m\left(D_{0}\right) f\left(D, D_{0}\right) g\left(D_{0}\right) \ln \left(D_{0}\right) \mathrm{d} D_{0}-g(D) n .
$$

The use of equation (1.2) requires the solution of three closure problems: the first involves the breakup frequency, $g(D)$, the second is the average number of daughter particles formed from the breakup of a mother particle of size $D_{0}, m\left(D_{0}\right)$ and the third is the p.d.f. of the daughter particles $f\left(D, D_{0}\right)$. Although equation (1.2) applies to either drops or bubbles, in this study, we will restrict our analysis to only the case of bubbles breaking in a turbulent water flow.

In Part 1 we have shown that under dilute conditions of air bubbles immersed in a turbulent water flow whose turbulence is locally homogeneous and nearly in equilibrium, the bubble's breakup frequency is a function of both its size and the turbulent kinetic energy (or the dissipation rate, $\epsilon$ ) of the underlying turbulence,

$$
g\left(\epsilon, D_{0}\right)=K_{\mathrm{g}} \frac{\sqrt{8.2\left(\epsilon D_{0}\right)^{2 / 3}-12 \sigma /\left(\rho D_{0}\right)}}{D_{0}},
$$

where $K_{\mathrm{g}}$ is a constant found experimentally to be equal to 0.25 . The above breakup frequency, which is based on the simple premise that the breakup time of the bubbles is inversely proportional to the sum of the non-inertial forces acting on its surface (resulting in deformation and confinement), was found to be in excellent agreement with recent experimental measurements (Part 1). In the present study, we will use this model for the bubble breakup frequency to solve the remaining two closure problems, i.e. to provide models for $m\left(D_{0}\right)$ and $f\left(D, D_{0}\right)$.

In the past, three main approaches have been used to model $f\left(D, D_{0}\right)$ : phenomenological models based on surface energy considerations (Tsouris \& Tavlarides 1994), statistical models (Coulaloglou \& Tavlarides 1977; Prince \& Blanch 1990; LonguetHiggins 1992; Novikov 1997), and hybrid models based on a combination of both 
(Konno et al. 1983; Cohen 1991). Among the most widely used phenomenological models based on surface energy considerations is the one proposed by Tsouris \& Tavlarides (1994). They proposed that upon breakup, $m\left(D_{0}\right)=2$, and only two daughter droplets of size $D_{1}$ and $D_{2}$ are formed whose most probable sizes are inversely proportional to the amount of surface energy created in the breakup process. This gives a minimum probability for the formation of two particles of the same size (since their surface energy is maximum), and a maximum probability for the formation of a pair made up of a very large particle and a complementary very small one. To avoid the singularity present in their model, they also assumed the existence of a minimum particle size, $D_{m i n}$. Their model for the daughter particle p.d.f. gives

$$
f\left(D_{1}, D_{0}\right)=\frac{e_{\min }+\left[e_{\max }-e\left(D_{1}\right)\right]}{\int_{0}^{D_{0}}\left(e_{\min }+\left[e_{\max }-e\left(D_{1}\right)\right]\right) \mathrm{d} D_{1}},
$$

where the energy to form a particle of size $D_{1}, e\left(D_{1}\right)$, is given by

$$
e\left(D_{1}\right)=\pi \sigma D_{1}^{2}+\pi \sigma D_{2}^{2}-\pi \sigma D_{0}^{2}=\pi \sigma D_{0}^{2}\left[\left(\frac{D_{1}}{D_{0}}\right)^{2}+\left[1-\left(\frac{D_{1}}{D_{0}}\right)^{3}\right]^{2 / 3}-1\right] .
$$

The maximum energy, $e_{\max }\left(D_{0}\right)$, corresponding to the formation of two particles of diameter $D_{1}=D_{2}=D_{0} / 2^{1 / 3}$ is

$$
e_{\max }=\pi \sigma D_{0}^{2}\left[2^{1 / 3}-1\right] .
$$

As indicated above, equation (1.5) reaches a minimum value when a particle of minimum diameter, $D_{\min }$, and a complementary one of maximum size, $D_{\max }=\left(D_{0}^{3}-\right.$ $\left.D_{\min }^{3}\right)^{1 / 3}$, are formed. The minimum energy, $e_{\min }$, is given by

$$
e_{\min }=\pi \sigma D_{0}^{2}\left[\left(\frac{D_{\min }}{D_{0}}\right)^{2}+\left[1-\left(\frac{D_{\min }}{D_{0}}\right)^{3}\right]^{2 / 3}-1\right] \text {. }
$$

The daughter particle's size p.d.f. given by equation (1.4) has a $U$ shape and has been shown to lead to results which are radically different from the experimental distributions measured in stirred tanks and other turbulent flows (Hesketh, Etchells \& Russell 1991; Sathyagal \& Ramkrishna 1996; Kostoglou \& Karabelas 1998; Part 1; and others). In addition, an important unresolved issue in the above model is the need to define a criterion for the value of $D_{\min }$.

Pure statistical distributions have also been used by Coulaloglou \& Tavlarides (1977), Prince \& Blanch (1990), and others. Coulaloglou \& Tavlarides also assumed that $m\left(D_{0}\right)=2$, and that the probability distribution function of daughter droplets (or bubbles) is well represented by a normal distribution (as proposed by Valentas \& Amudson 1966). By selecting the variance so that more than $99.6 \%$ of the daughter droplets were in the range of volumes between 0 and $v_{0}=\pi D_{0}^{3} / 6$, they obtained a daughter particle size p.d.f. as

$$
f\left(v, v_{0}\right)=\frac{2.4}{v_{0}} \exp \left[-4.5 \frac{\left(2 v-v_{0}\right)^{2}}{v_{0}^{2}}\right],
$$

where $v_{0}$ and $v$ are the volumes of the mother and daughter particles respectively. Coulaloglou \& Tavlarides showed that this distribution could be adjusted to predict some of the experimental results obtained in stirred tanks. 
Purely statistical studies have been performed by Longuet-Higgins (1992), Novikov (1997), and others. Longuet-Higgins proposed a simple mechanism for the breakup process by simulating it through a series of a random divisions of a cubical block of size unity by a number of planes which are parallel to the faces of the block. In studying the p.d.f. resulting from cuts performed in one, two, and three dimensions, he obtained an infinite number of possible distributions depending on the number of random partitions performed in the initial dimension. By adjusting the combination of the number of cuts performed in each dimension, he showed that the model could fit various experimental results. In the Appendix Longuet-Higgins compares some of the measurements described in Part 1 with this model.

Concerning hybrid models, it is worth noting the model proposed by Konno $\mathrm{et} \mathrm{al}$. $(1980,1983)$. This model is based on their experimental observations that three daughter droplets are produced from the breakup of a mother one, $m\left(D_{0}\right)=3$. They calculated the probability of obtaining a certain combination of three droplets and weighted their probability by a factor proportional to the energy contained in the turbulent scales of sizes equal to the size of the daughter droplets. They assumed that the volume of a mother droplet, $v_{0}$, is divided into $J$ units of volume elements $v_{e}$, so that $J=v_{0} / v_{e}$. Their statistical model then states that the mother droplet breaks into $m$ daughter droplets whose volume is given by a random combination of elements $v_{e}$. The number of elements of each daughter droplet formed, numbered by the index $i$, is given by $K_{i}=$ $v_{i} / v_{e}$, where $v_{i}$ is the volume of the droplet $i$. All combinations are then derived from the different arrangements of $K_{1}, K_{2}, \ldots, K_{m}$, which satisfy the conservation of volume,

$$
K_{1}+K_{2}+\cdots+K_{m}=J
$$

The combinations of the $J$ elements, taken in $m$ groups satisfying equation (1.9), gives all the possible values of the non-dimensional combinations of volumes $K_{i}$ that can be formed. The probability of a certain combination of $K_{i}$ elements is then given by

$$
P \propto E\left(K_{1}\right) E\left(K_{2}\right) E\left(K_{3}\right) \ldots E\left(K_{m}\right),
$$

where $E\left(K_{i}\right)$ is the spectrum function of the turbulent kinetic energy estimated from the Heisenberg energy spectrum as

$$
E\left(K_{i}\right)=\left(\frac{8}{9 \alpha}\right)^{2 / 3} \epsilon^{2 / 3}\left(\frac{\pi}{6 v_{e} K_{i}}\right)^{-5 / 3}\left(1+\frac{8 v^{3}\left(\pi / 6 v_{e} K_{i}\right)^{4}}{3 \alpha^{2}}\right)^{-4 / 3},
$$

where $\alpha=0.51$, and $v$ is the kinematic viscosity of the continuous phase.

To obtain a continuous distribution with this model, one needs to use a large value for $J$, and Konno et al. used $J=100$. Konno's distribution produces a low probability for combinations of very large and very small particles and gives a maximum probability for combinations of particles of similar sizes. This model has the deficiency that the distribution of the daughter droplets is basically neither dependent on the size of the mother droplets, nor on the turbulent kinetic energy of the underlying turbulence, two facts which are contrary to experimental observations. In fact. Konno's distribution is practically a universal one, and can be approximated by a Beta function (Konno et al. 1983):

$$
D_{0} f\left(D, D_{0}\right)=f^{*}\left(D^{*}\right)=\frac{\Gamma(12)}{\Gamma(9) \Gamma(3)}\left(\frac{D}{D_{0}}\right)^{8}\left(1-\frac{D}{D_{0}}\right)^{2},
$$

where $f^{*}\left(D^{*}\right)$ represents the probability density function of the daughter particles 
resulting from the breakup of a mother particle of size $D_{0}$ using a non-dimensional diameter of the daughter particle defined as $D^{*}=D / D_{0}$.

Another hybrid model based on energy and entropy considerations has been proposed by Cohen (1991). Using entropy arguments, he found that the most probable distribution resulting from the shattering of a mother droplet is similar to the Beta function proposed by Konno et al. (1983). However, his p.d.f. model incorporated a dependence on $\epsilon$. Although Cohen's model is an elegant one, it has the drawback of producing an explosive breakup in which a droplet breaks into a very large number of daughter droplets (several thousands), which has not been observed in any experiments.

In this paper we will use a detailed set of measurements of the transient evolution of $n(D, x, t)$, performed over a wide range of bubble sizes and turbulent kinetic energy of the underlying turbulence to test a new phenomenological model for $f\left(D, D_{0}\right)$. These measurements, first reported in Part 1, were conducted by measuring the transient bubble-size p.d.f. resulting from the breakup of a volume of air of a certain size injected into the fully developed region along the axis of a high-Reynolds-number water jet. Rather than attempting to solve the otherwise intractable inverse problem of calculating $f\left(D, D_{0}\right)$ from equation (1.2), we propose a new phenomenological model for the daughter bubble-size p.d.f. based on a weighted probability model. This model is then contrasted to previous ones and compared to the experimental measurements.

In $\S 2$ we formulate the phenomenological model for the daughter bubble size p.d.f. This model is then compared to available experimental evidence and to previously formulated theories in $\$ 3$. Finally, some concluding remarks are presented in $\$ 4$.

\section{Model formulation}

Consider a mother or parent bubble (mother or parent refers to a bubble before breakup) to be spherical with an initial diameter $D_{0}$. At time $t=t_{0}$, the bubble is immersed in a stationary water flow whose turbulence is nearly homogeneous and isotropic. The initial bubble diameter is assumed to be in the inertial subrange of the underlying turbulence, $\eta \ll D_{0} \ll L_{x}$, where $\eta$ is the Kolmogorov microscale of the viscous dissipation, and $L_{x}$ is the integral length scale.

When the air bubble is injected into the turbulent water jet, the velocity fluctuations of the underlying turbulence result in pressure deformation forces acting on the bubble's surface that, when greater than the confinement forces due to surface tension, will cause its breakup. Since the Ohnesorge numbers of the bubbles of interest here are always very small $\left(O h=\mu_{a} / \sqrt{\rho_{a} \sigma D}<10^{-3}\right)$, the internal viscous deformation forces are negligible compared to the surface tension forces, and thus will be ignored.

The average deformation energy per unit volume acting on the surface of the bubbles is

$$
\tau_{t}=\frac{1}{2} \rho \beta \epsilon^{2 / 3} D_{0}^{2 / 3},
$$

where $\beta=8.2$ is a constant obtained by integrating the difference between the velocity fluctuations, $\overline{\Delta u^{2}}\left(D_{0}\right)$, of two points separated by a distance $D_{0}$. over the whole range of turbulence scales, Batchelor (1956). The confinement energy per unit volume is simply

$$
\tau_{s}=\frac{6 \pi \sigma D_{0}^{2}}{\pi D_{0}^{3}}=6 \frac{\sigma}{D_{0}} .
$$

When $\tau_{t}>\tau_{s}$ the bubble will break in a certain time $t_{b}$, with a frequency $g\left(\epsilon, D_{0}\right)=1 / t_{b}$. 


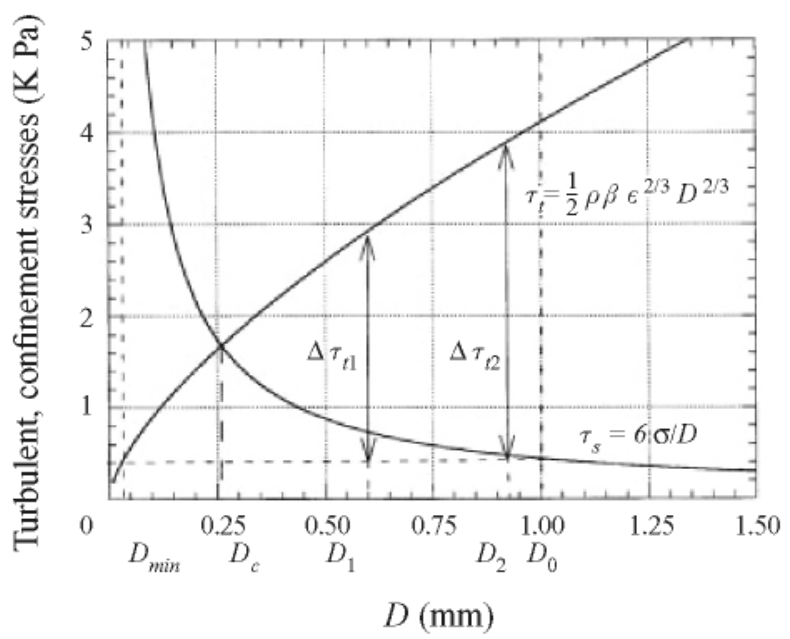

FIGURE 1. Difference in stresses associated with the formation of a bubble, $D_{1}$, and its complement, $D_{2}, \Delta \tau_{t 1}=\frac{1}{2} \rho \beta\left(\epsilon D_{1}\right)^{2 / 3}-6 \sigma / D_{0}$ and $\Delta \tau_{t 2}=\frac{1}{2} \rho \beta\left(\epsilon D_{2}\right)^{2 / 3}-6 \sigma / D_{0}$. In this example, the mother bubble is of size $D_{0}=1 \mathrm{~mm}$, and $\epsilon=1000 \mathrm{~m}^{2} \mathrm{~s}^{-3}$.

In Part 1 , we have shown that the breakup time of a bubble of size $D_{0}$ can be estimated from the deformation acceleration, $a_{b}=u_{b} / t_{b}$, produced by the non-inertial forces acting on the surface of the bubble. Here, $u_{b}$ is the deformation velocity, and $t_{b}$ is the bubble's breakup time given by

$$
t_{b} \propto \frac{D_{0}}{\sqrt{\beta\left(\epsilon D_{0}\right)^{2 / 3}-12 \sigma /\left(\rho D_{0}\right)}} .
$$

As shown in the above equation, the breakup frequency, $g\left(\epsilon, D_{0}\right)=1 / t_{b}$, depends on both the size of the bubble and the value of the turbulent kinetic energy (or dissipation rate, $\epsilon$ ) of the underlying turbulence.

Upon breakup, the bubble is shattered into an array of smaller bubbles whose size p.d.f. is denoted by $f\left(D, D_{0}\right)$. The first premise of our bubble shattering model is that upon breakage, a bubble of size $D_{0}$ only breaks into two bubbles $\left[m\left(D_{0}\right)=2\right]$ of complementary masses with diameters $D_{1}$ and $D_{2}$ (an important point to be addressed later on). If the stochastic variable $D_{1}$ were uniformly distributed on the segment $\left[0, D_{0}\right], D^{*}=D_{1} / D_{0}$ would be uniformly distributed on the segment $[0,1]$ and its probability density would always be $p\left(D^{*}\right)=1$ (Longuet-Higgins 1992). However, since the values of the pressure deformation forces, $\tau_{t}$, are not uniform with the distance $D_{1}$, our bubble splitting phenomenon cannot produce a uniformly distributed density function. In fact, for a spherical bubble of size $D_{0}$, there is a minimum distance $D_{\min }$, such that the turbulent stresses acting between two points separated by this distance, $\frac{1}{2} \rho \beta\left(\epsilon D_{\min }\right)^{2 / 3}$, are just equal to the confinement pressure due to the surface tension, $6 \sigma / D_{0}$ (see figure 1). Thus, the probability of the splitting of a fraction of size $D_{1}<D_{\min }=12 \sigma /\left(\beta \rho D_{0}\right)^{3 / 2} \epsilon^{-1}$, from a bubble of size $D_{0}$ should be zero. On the other hand, it appears reasonable to accept that the probability of the splitting of any portion of size $D_{\min }<D_{1}<D_{0}$ should be weighted by the difference in the stresses, $\Delta \tau_{t 1}=\frac{1}{2} \rho \beta\left(\epsilon D_{1}\right)^{2 / 3}-6 \sigma / D_{0}$, associated with the formation of a bubble of size $D_{1}$. However, associated with the generation of a bubble of size $D_{1}$, there is also the formation of a complementary bubble of size $D_{2}=D_{0}\left[1-\left(D_{1} / D_{0}\right)^{3}\right]^{1 / 3}$, involving 


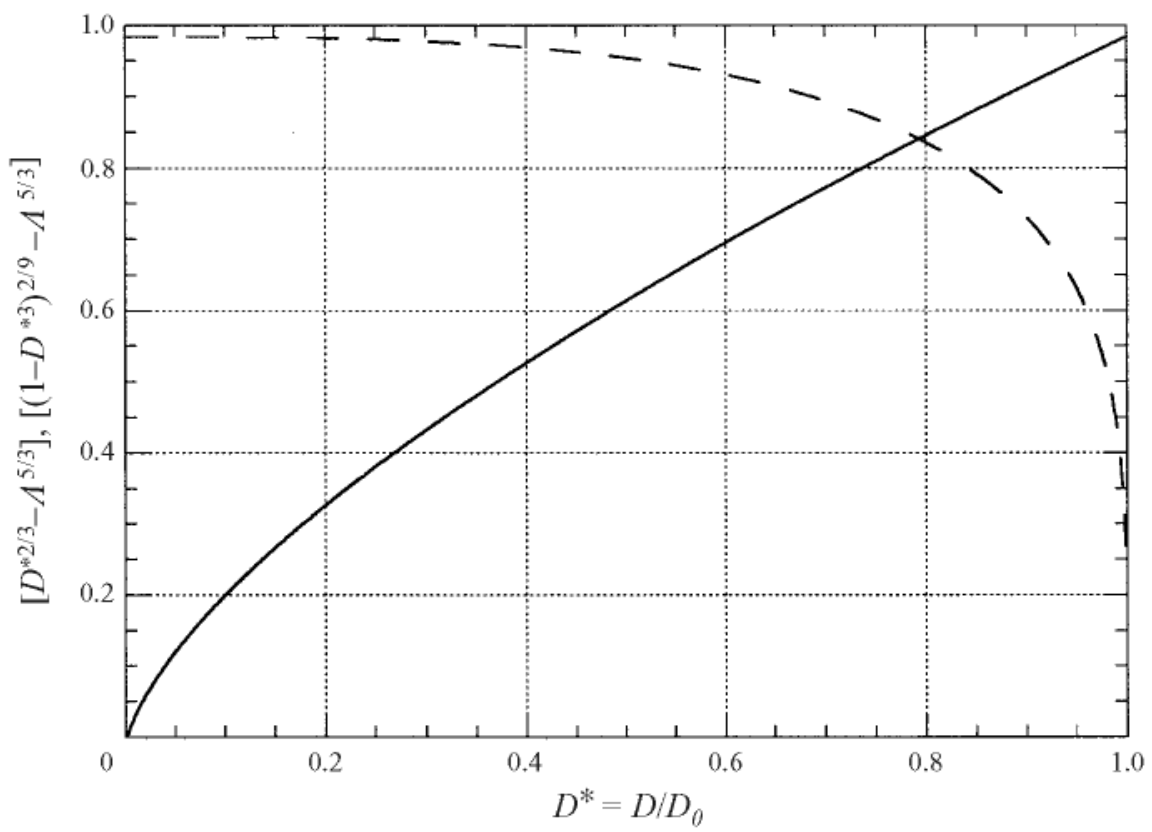

FIGURE 2. Difference in stresses associated with the formation of a bubble (solid line) and its complement (dashed line) given in equation (2.6). In this particular case, the mother bubble is of size $D_{0}=3 \mathrm{~mm}$, and the dissipation rate is $\epsilon=1000 \mathrm{~m}^{2} \mathrm{~s}^{-3}$.

a difference of stresses $\Delta \tau_{t 2}=\frac{1}{2} \rho \beta\left(\epsilon D_{2}\right)^{2 / 3}-6 \sigma / D_{0}$. Thus, we postulate that the probability of the formation of a pair of bubbles of sizes $D_{1}$ and $D_{2}$ from the shattering of a mother bubble of size $D_{0}$ is weighted by the product of the two surplus stresses,

$$
\left.\begin{array}{ll}
P\left(D^{*}\right)=0 & \text { for } D^{*}<D_{\min }^{*}, \\
P\left(D^{*}\right) \propto p\left(D^{*}\right)\left[\Delta \tau_{t 1}\right]\left[\Delta \tau_{t 2}\right] & \text { for } D_{\min }^{*} \leqslant D^{*} \leqslant D_{\max }^{*}, \\
P\left(D^{*}\right)=0 & \text { for } D^{*}>D_{\max }^{*},
\end{array}\right\}
$$

or similarly,

$$
P\left(D^{*}\right) \propto\left[\frac{1}{2} \rho \beta\left(\epsilon D^{*} D_{0}\right)^{2 / 3}-\frac{6 \sigma}{D_{0}}\right]\left[\frac{1}{2} \rho \beta\left(\epsilon D_{2}^{*} D_{0}\right)^{2 / 3}-\frac{6 \sigma}{D_{0}}\right],
$$

in the range $D_{\text {min }}^{*} \leqslant D^{*} \leqslant D_{\text {max }}^{*}$ where $D^{*}=D_{1} / D_{0}$ and $D_{\text {min }}^{*}=D_{\min } / D_{0}=(12 \sigma /(\beta \rho))^{3 / 2}$ $D_{0}^{-5 / 2} \epsilon^{-1}$. In other words, we postulate that the probability of a certain sized pair to form should be weighted by the product of the excess stresses associated with the length scales corresponding to each bubble size. From a simple mass balance, and neglecting the compressibility effect in the air inside the bubbles, $D_{2}=D_{0}\left[1-\left(D_{1} / D_{0}\right)^{3}\right]^{1 / 3}$, and

$$
P\left(D^{*}\right) \propto\left(\frac{1}{2} \rho \beta\left(\epsilon D_{0}\right)^{2 / 3}\right)^{2}\left[D^{* 2 / 3}-\Lambda^{5 / 3}\right]\left[\left(1-D^{* 3}\right)^{2 / 9}-\Lambda^{5 / 3}\right],
$$

where $A=D_{c} / D_{0}$, and $D_{c}=(12 \sigma /(\beta \rho))^{3 / 5} \epsilon^{-2 / 5}$ is a critical diameter defined by the crossing point of the curves $\tau_{t}$ and $\tau_{s}$ in figure 1 . Our model implies that $D_{\min }^{*} \leqslant D^{*} \leqslant$ $D_{\max }^{*}$ or, equivalently, that $D_{\min } \leqslant D_{1} \leqslant D_{\max }$ where $D_{\min }=\left(12 \sigma /\left(\beta \rho D_{0}\right)\right)^{3 / 2} \epsilon^{-1}$ and $D_{\max }=D_{0}\left[1-\left(D_{\min } / D_{0}\right)^{3}\right]^{1 / 3}$, provided that $D_{\min }>\eta$. Otherwise, $D_{\min }$ is taken to be equal to $\eta$. 

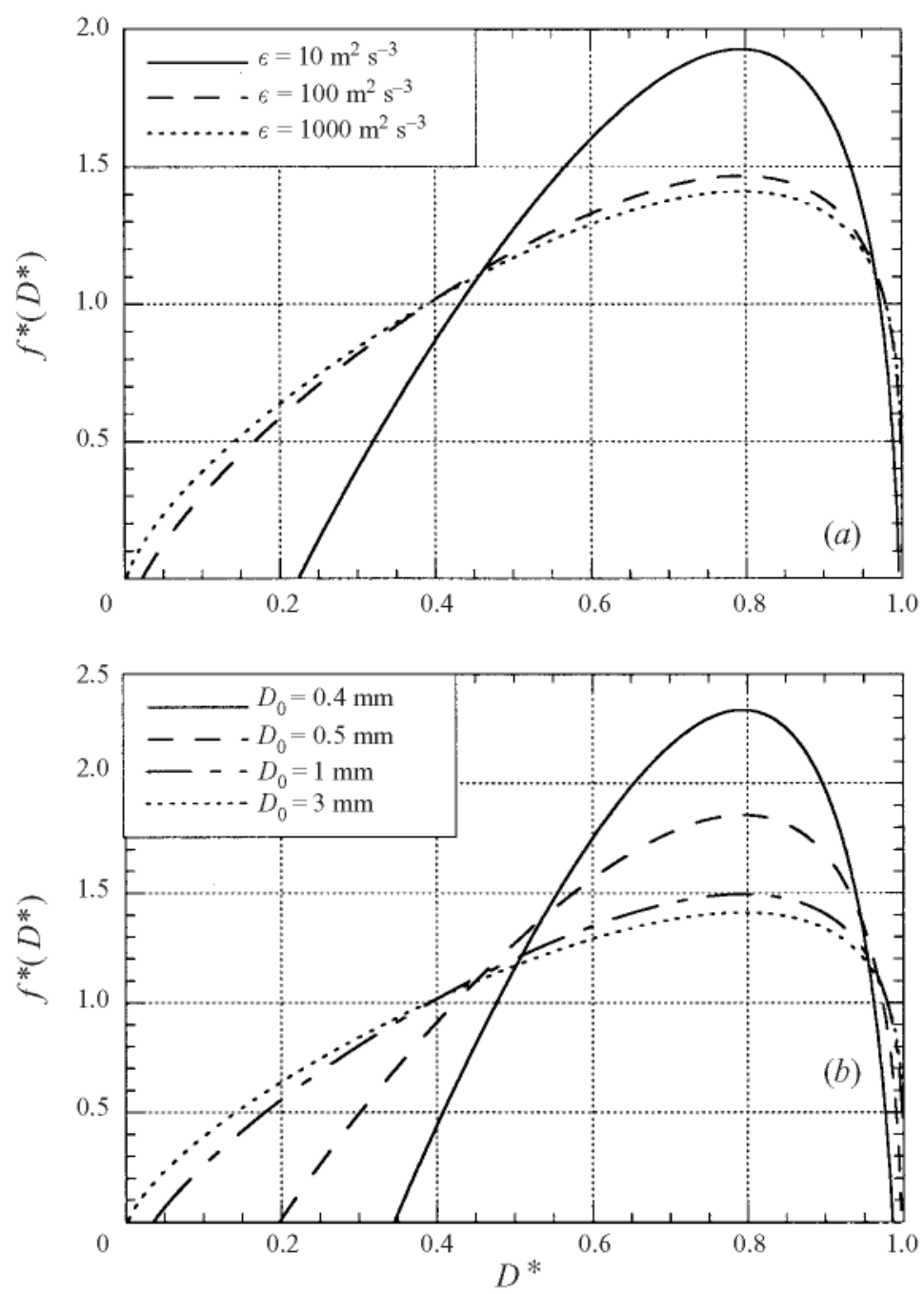

FIGURE 3. Probability density functions of the daughter bubbles formed from the breakup of a mother one of size $D_{0}$. (a) Evolution of the p.d.f. for various values of $\epsilon$ and fixed $D_{0}=3 \mathrm{~mm}$. (b) Influence of $D_{0}$ on the p.d.f. for a fixed value of $\epsilon=1000 \mathrm{~m}^{2} \mathrm{~s}^{-3}$.

The difference in stresses associated with the formation of a bubble and its complement given in equation (2.6) are shown in figure 2. Using the normalization condition of the density of probability, $\int_{0}^{1} f^{*}\left(D^{*}\right) \mathrm{d}\left(D^{*}\right)=1$, the daughter probability density function of $D^{*}, f^{*}\left(D^{*}\right)$, can then be written as

$$
f^{*}\left(D^{*}\right)=\frac{P\left(D^{*}\right)}{\int_{0}^{1} P\left(D^{*}\right)}=\frac{\left[D^{* 2 / 3}-\Lambda^{5 / 3}\right]\left[\left(1-D^{* 3}\right)^{2 / 9}-\Lambda^{5 / 3}\right]}{\int_{D_{\min }^{*}}^{D_{\max }^{*}}\left[D^{* 2 / 3}-\Lambda^{5 / 3}\right]\left[\left(1-D^{* 3}\right)^{2 / 9}-\Lambda^{5 / 3}\right] \mathrm{d}\left(D^{*}\right)} .
$$

The probability density function of the daughter bubbles resulting from the breakup of a mother bubble, $D_{0}$, is then calculated as $f\left(D_{1}, D_{0}\right)=f^{*}\left(D^{*}\right) / D_{0}$. The dependence of $f^{*}\left(D^{*}\right)$ on $\epsilon$ and $D_{0}$ is shown in figure 3. Note that the peak of the distribution is 
always located at $D^{*}=\left(\frac{1}{2}\right)^{1 / 3} \simeq 0.8$, which corresponds to the case of two daughter bubbles with the same volume, and that the p.d.f.s become wider as either $\epsilon$ or $D_{0}$ are increased.

It should first be made clear that we are proposing a statistical model. Clearly our phenomenological model represents a simplified view of the bubble by assuming sphericity. However, we believe that this assumption does not limit its validity. The confinement energy per unit volume is a minimum if the bubble is spherical, and the minimum necessary energy to deform the bubble is inversely proportional to this spherical diameter. We then postulate that the probability of a certain breakup occurring should be proportional to the difference between the deformation energy due to the turbulence and this minimum confinement value (if the bubbles are not spherical the confinement energy should be even larger).

\section{Experimental results and discussion}

A detailed description of the experimental facility and measurement techniques used in the experiments reported here can be found in Part $\mathbf{1}$, and in Martínez-Bazan (1998). The reader is referred to these two publications for details. For the purpose of our discussion, in this section we will simply summarize the most salient features of these experiments.

The experimental facility used to perform the experiments on the breakup of a bubble in a turbulent water flow consists of a submerged turbulent water jet where a continuous air jet is injected through a small-diameter hypodermic needle located at a given position along the central axis in the fully developed turbulent region of the jet. This allowed us to assume that the underlying turbulence where the breakup takes place is nearly homogeneous and isotropic in the absence of any moving solid surfaces. The evolution of the bubble size p.d.f., as the bubbles are broken by the turbulence and transported downstream by the mean convective motion of the jet, was measured using digital image processing techniques. These bubble size p.d.f. measurements were then discretized into 150 size bins and used to calculate the rate of change with respect to position of the number of bubbles of a certain size per unit volume as the bubble cloud was convected into regions of decreasing values of $\epsilon$. Several sets of experiments were performed in which both the initial bubble size and the initial value of $\epsilon$ at the injection point were systematically changed. A summary of the test conditions used for the measurements is given in table 1 in Part 1.

We will begin by discussing the results of Set 2 in table 1 in Part 1 . In this case, the air was injected on the jet's centreline at a location 15 jet diameters downstream from the jet's exit nozzle. The initial jet velocity was $U_{0}=17 \mathrm{~ms}^{-1}$, and the jet's Reynolds number was $R_{e}=U_{0} D_{j}^{*} / v=51000$. The air injection velocity was $U_{a}=9.84 \mathrm{~m} \mathrm{~s}^{-1}$, and equal to the local mean velocity of the water at the air injection point. Thus, upon injection into the water, the air bubbles were only subjected to the velocity fluctuations of the underlying turbulence of the water flow. These turbulent stresses resulted in deformation forces that were much greater than the confinement forces due to surface tension, and the bubble was observed breaking into a set of daughter ones. Since the characteristic breakup time of the bubble depends on its size, $D_{0}$, and on the value of the turbulent kinetic energy (or dissipation rate, $\epsilon$ ) of the underlying turbulence, this breakup process continued while the resulting array of bubbles was convected downstream to regions of lower and lower dissipation rate, $\epsilon$, until it was observed that the bubble size p.d.f. eventually reached a frozen or unchanged shape (Part 1). 

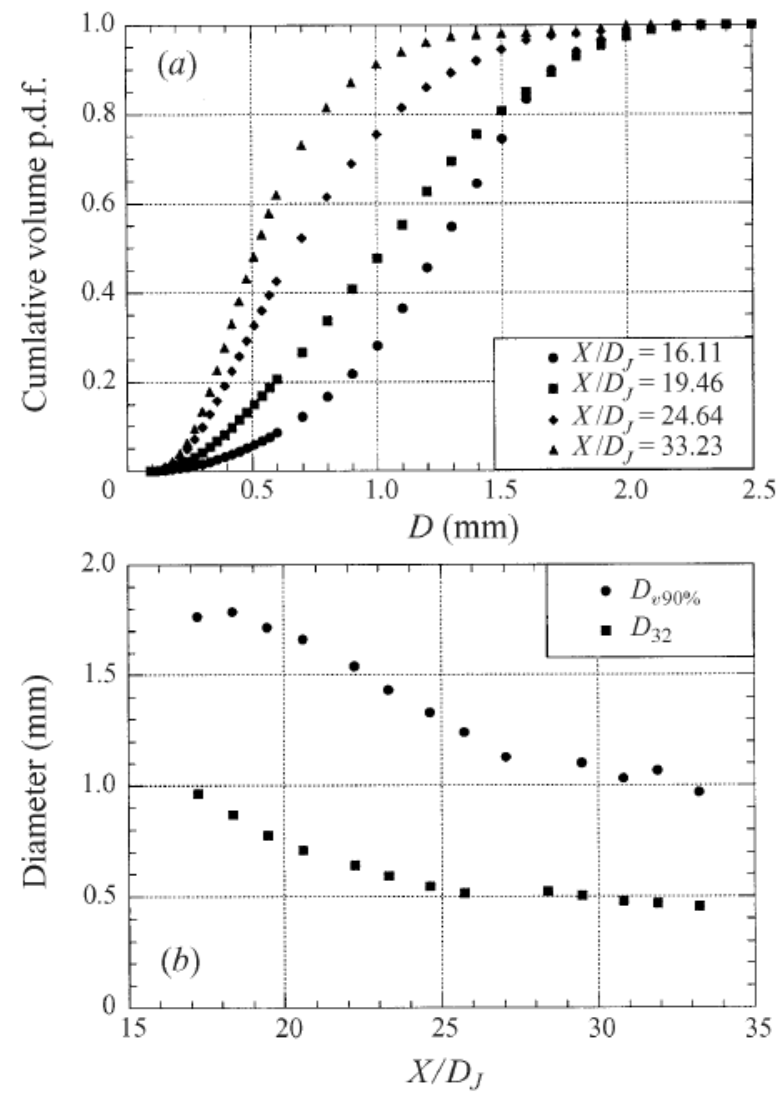

FIGURE 4. (a) Downstream evolution of the cumulative volume probability density function (b) Downstream evolution of the Sauter mean diameter, $D_{32}=\sum N_{i} D_{i}^{3} / \sum N_{i} D_{i}^{2}$ and $D_{v 90 \%}$. Experimental Set 2.

The downstream evolution of the cumulative bubble volume p.d.f. corresponding to this experiment is shown in figure 4(a) where, for clarity, we have presented four measuring locations only. Note that all the values of the characteristics diameters and the various moments of the size distribution, i.e. $D_{v 90 \%}$ and $D_{32} \dagger$, decay with the downstream distance, $X / D_{J}$, due to the breakup process until they all reach asymptotic values at a downstream distance of approximately $28 D_{J}$, at which point the cumulative volume p.d.f. is no longer observed to change.

It is important to emphasize that in all our experiments the bubbles broke up under the action of a fully developed, spatially nearly uniform, isotropic turbulence, and in the absence of any solid surfaces. The air was always injected at the jet's centre axis, and during their breakup, the bubbles remained at the centre of the jet, being transported laterally by the action of the turbulence to radial distances always smaller than $30 \%$ of the width of the jet. Thus, the process can be assumed to be one-dimensional. The radial dispersion did not affect the measurements, and indeed, we measured only the bubble size p.d.f. resulting from the breakup and convective

$\dagger D_{32}=\sum N_{i} D_{i}^{3} / \sum N_{i} D_{i}^{2}, D_{10}=\sum N_{i} D_{i} / \sum N_{i}$ where $N_{i}$ is the number of bubbles of size $D_{i}$ measured. $D_{v 90 \%}=$ diameter of a bubble such that $90 \%$ of the total volume of air is contained in bubbles of smaller diameter. 
transport at each downstream location. As mentioned above, coalescence effects were negligibly small in our experiments since the bubble void fraction was always $<10^{-5}$.

\subsection{Comparison of the theoretical model with the experimental results}

The phenomenological model for the daughter p.d.f. presented in $\$ 2$ was used to solve equation (1.2) in order to obtain the evolutions of the bubble size distributions and to compare them with the above experimental results. Since the problem of interest here is steady state, $\partial n / \partial t=0$, equation (1.2) reduces to

$$
\frac{\partial(U n)}{\partial x}=\int_{D}^{\infty} m\left(D_{0}\right) f\left(D, D_{0}\right) g\left(D_{0}\right) n\left(D_{0}\right) \mathrm{d} D_{0}-g(D) n,
$$

where $U(x)$ is the mean centreline velocity of the water jet, which is equal to the mean convective velocity of all bubbles $v(D, x, t)$ regardless of their size $D$.

In the steady-state experiments described above our measurements show that all the bubbles, regardless of their size, are convected at the same mean velocity, equal to the mean local velocity of the water jet (Martínez-Bazán 1998). Thus, the term $\partial(U n) / \partial x$ in equation (3.1) is simply $\partial(U(x) n) / \partial x$, where $U(x)$ is now the corresponding mean velocity of the water jet measured at each measurement location, and $x$ is the downstream location along the axis of the jet, i.e. this term represents the spatial downstream evolution of the number density of the bubbles of size $D$ as they are transported downstream by the convective velocity of the water jet. When integrating the right-hand side of equation (3.1) we used the value of $g\left(\epsilon, D_{0}\right)$ calculated from equation (1.3), and the experimentally measured values of $\epsilon$ (figure 3 in Part 1) at each downstream location. The initial conditions used to integrate equation (3.1) were taken to be equal to the size p.d.f. measured at the first measurement location. $X / D_{J}=16.1$.

The downstream evolution of the cumulative bubble volume p.d.f. resulting from the above calculation performed by integrating equation (3.1) is given in figures $5(a)$ and $5(b)$, where it is compared to the experimental measurements. Note that the agreement with the experimental data is very good, not only concerning the various moments of the bubble size distribution (figure $5 b$ ), but more importantly, between the shape of the measured and the calculated cumulative volume p.d.f.s (figure $5 a$ ). The same excellent agreement shown between the calculated and the measured p.d.f.s was also found for the other cases studied (see figures $6 a$ and $6 b$ corresponding to Set 3a, Part 1).

We will now turn our attention to discussing the assumption of binary splitting. Our model is based on the assumption that upon breaking, the bubble is broken into two daughter ones, $m\left(D_{0}\right)=2$. This appears to be consistent with all of our measurements performed at low and moderate values of the turbulent Weber number, $W e_{t}=\rho \overline{\Delta u^{2}}\left(D_{0}\right) D_{0} / \sigma$. In these cases, the surface of the mother bubbles is always observed to be relatively smooth. A typical sequence of the evolution of a bubble during its breakup is shown in figure 7 . This sequence was taken at 6000 frames per second and shows consecutive steps in the bubble breakup process. Observe that the bubble marked $A_{1}$ breaks into two labelled $A_{11}$ and $A_{12}$. Subsequently $A_{11}$ breaks into $A_{111}$ and $A_{112}$, and later on the bubble $A_{12}$ breaks into $A_{121}$ and $A_{122}$. This binary splitting process appears to continue until the breakup is fully finished. However, although difficult to characterize, we have also observed that at larger values of the turbulent Weber number, the mean number of daughter bubbles, $m\left(D_{0}\right)$, increases. Thus, the assumption of binary breakup appears to be well justified only at low and moderate $W e_{t}$. Regardless of the weakness in the assumption of $m\left(D_{0}\right)=2$, the reason 

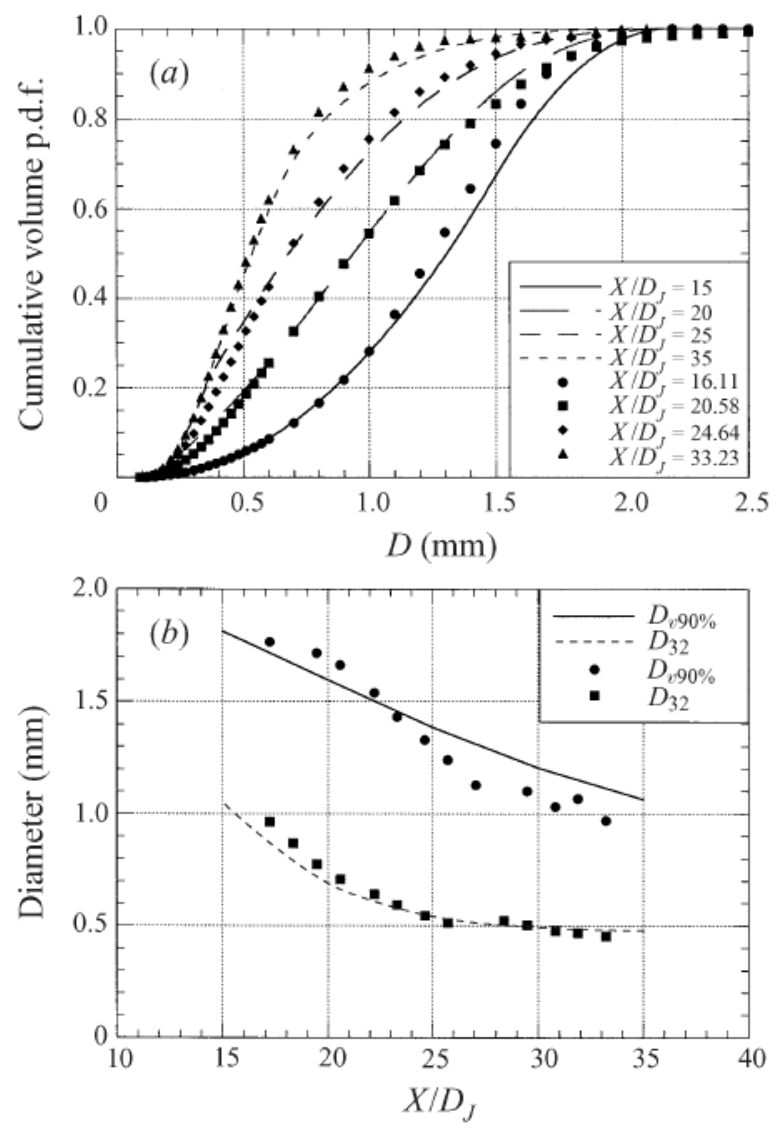

Figure 5. (a) Downstream evolution of the cumulative volume probability density function. (b) Downstream evolution of the Sauter mean diameter, $D_{32}$, and $D_{v 90 \%}$. Initial value of the dissipation rate of TKE was $\epsilon_{0}=2000 \mathrm{~m}^{2} \mathrm{~s}^{-3}$ at the air injection point, $X / D_{J}=15$. The lines represent the results obtained from the model integrating equation (3.1) and the symbols are the experimental measurements. Experimental Set 2.

for the excellent agreement observed between the measurements and the model is also due to the fact that the shape of the cumulative volume p.d.f. is fairly insensitive to the appearance of satellite small bubbles. This is a consequence of the fact that the volume fraction contained in the small bubbles is very small. Nevertheless, in most diffusion-controlled processes, the important parameter controlling the process is the Sauter Mean Diameter, $D_{32}$, which is an indication of the volume-to-surface ratio of the distribution function, and this is always very well predicted with the binary breakup assumption, as shown in figures $5(b)$ and $6(b)$.

At larger values of the Weber number, the binary breakup assumption needs to be revised. To address the possible formation of a larger number of daughter bubbles, we can use a simple estimate based on energy arguments. If the energy associated with the difference of turbulent velocities existing between two points separated a distance equal to the size of the bubbles were the only energy causing the changes in the energy associated to surface tension, and neglecting viscous and compressibility effects, the total energy associated with the differences of velocity and surface tension 

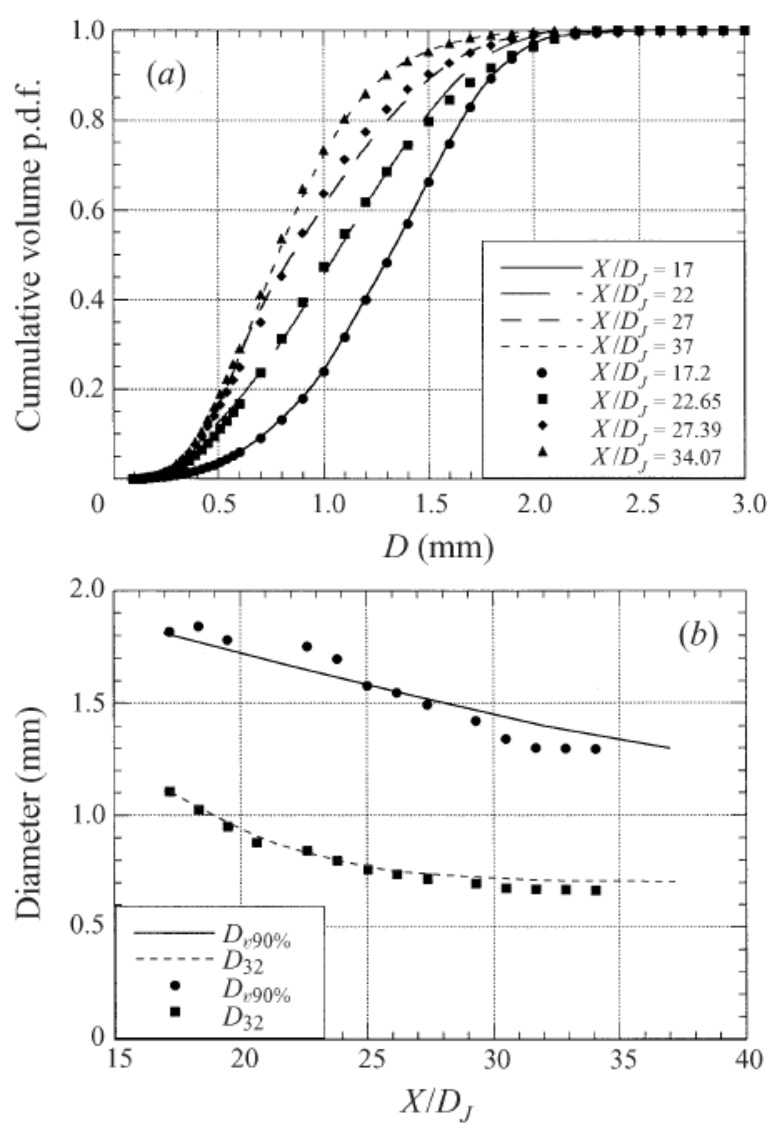

FIgURE 6. As figure 5 but for experimental Set $3 \mathrm{a}: \epsilon_{0}=1000 \mathrm{~m}^{2} \mathrm{~s}^{-3}$.

should remain constant during the breakup process. Thus,

$$
\frac{1}{2} \rho \beta\left(\epsilon D_{0}\right)^{2 / 3} \frac{1}{6} \pi D_{0}^{3}+\pi \sigma D_{0}^{2}=\sum_{i=1} \frac{1}{2} \rho \beta N_{i}\left(\epsilon D_{i}\right)^{2 / 3} \frac{1}{6} \pi D_{i}^{3}+\pi \sigma \sum_{i=1} N_{i} D_{i}^{2},
$$

where $N_{i}$ is the number of bubbles of size $D_{i}$, and $\sum_{i=1} N_{i} D_{i}^{3}=D_{0}^{3}$. Equation (3.2) can be rewritten as

$$
1+A=A \sum_{i=1} N_{i} D_{i}^{* 11 / 3}+\sum_{i=1} N_{i} D_{i}^{* 2}
$$

where $A=(\rho / 12 \sigma) \epsilon^{2 / 3} D_{0}^{5 / 3}=\Lambda^{-5 / 3}$, and $D^{*}=D / D_{0}$ is a non-dimensional diameter of the resulting daughter bubble. Expressing equation (3.3) as a function of the daughter bubble p.d.f., $f^{*}\left(D^{*}\right)$ :

$$
1+A=A N_{t} \int_{0}^{1} f^{*}\left(D^{*}\right) D^{* 11 / 3} \mathrm{~d} D^{*}+N_{t} \int_{0}^{1} f^{*}\left(D^{*}\right) D^{* 2} \mathrm{~d} D^{*}
$$

From conservation of volume,

$$
\frac{1}{N_{t}}=\int_{0}^{1} f^{*}\left(D^{*}\right) D^{* 3} \mathrm{~d} D^{*}=D_{30}^{* 3}
$$

Equations (3.4) and (3.5) can be solved by a trial and error scheme to obtain the 


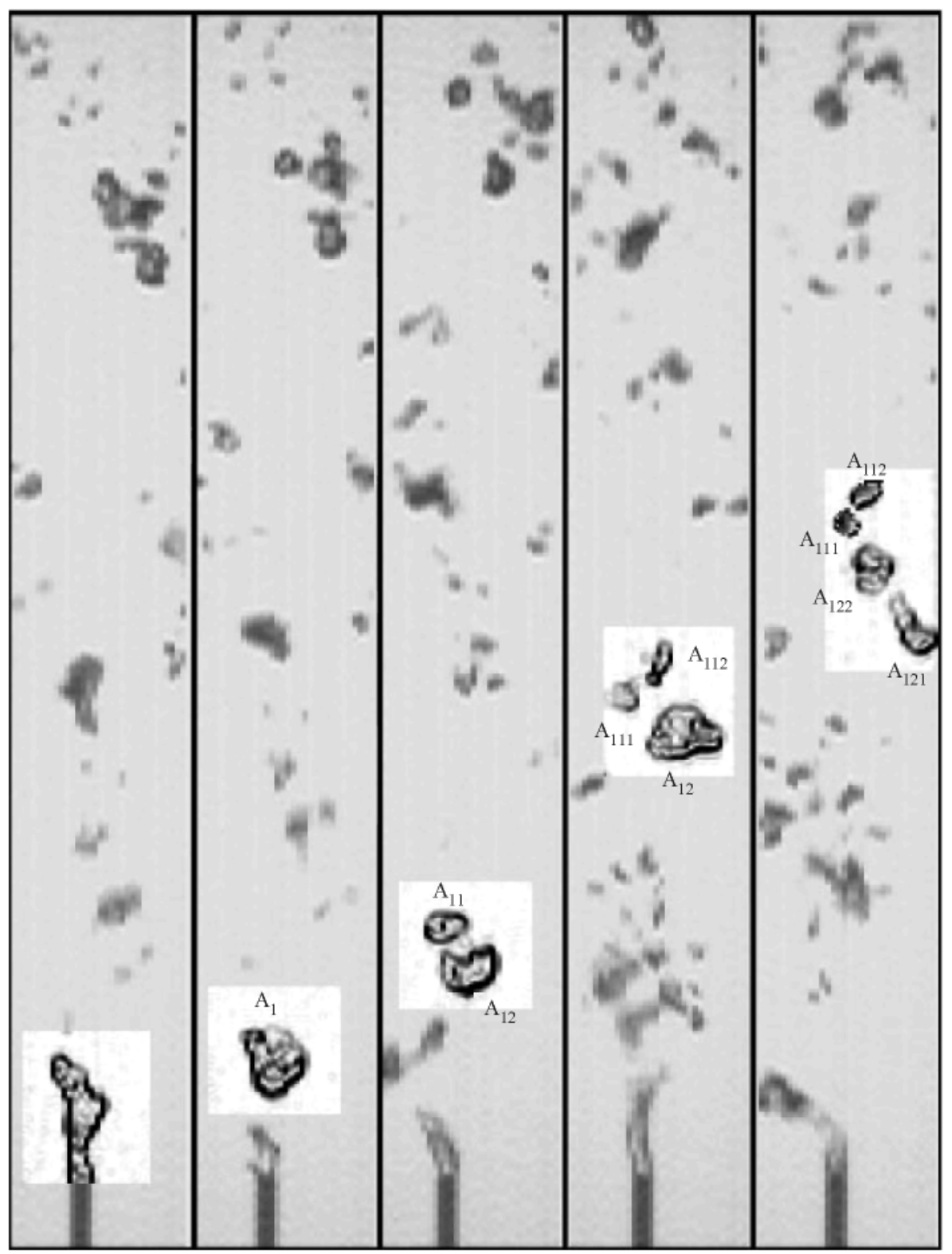

FIGURE 7. Time evolution of the characteristic breakup of a mother bubble showing a binary bubble splitting mechanism. The images were taken at 6000 frames per second.

number of bubbles resulting from the breakup of a mother bubble $D_{0}$ and the daughter-bubble p.d.f., $f^{*}\left(D^{*}\right)$, given in equation (2.7).

For the particular case of $\epsilon=1000 \mathrm{~m}^{2} \mathrm{~s}^{-3}$ and $D_{0}=2 \mathrm{~mm}$, as in the experimental Set 3c, we obtain $A=29.7$. For such value of $A$, substituting the daughter bubble p.d.f., $f^{*}\left(D^{*}\right)$, predicted by our model given in equation (2.7) into equations (3.4) and (3.5), the number of daughter-bubble obtained, for the same value of $A$, is approximately 3. Now, we can use this estimated number of daughter bubble to recalculate the 

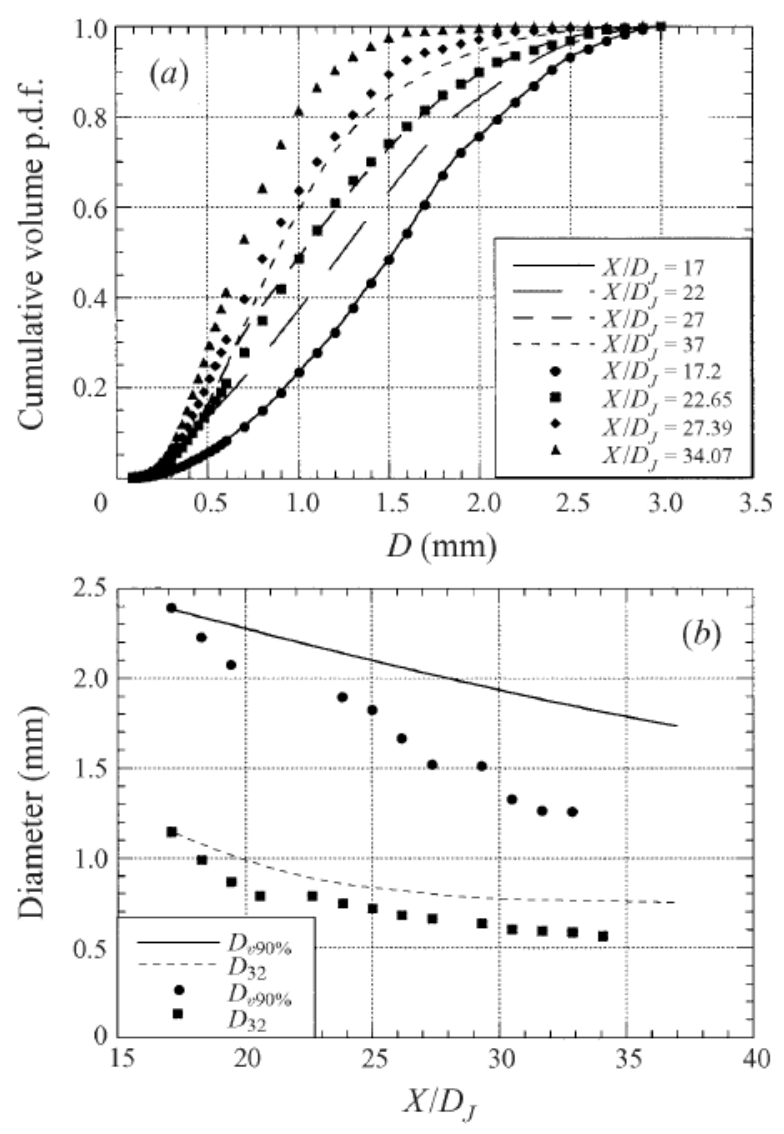

FIGURE 8. (a) Downstream evolution of the cumulative volume probability density function. (b) Downstream evolution of the Sauter mean diameter, $D_{32}$ and $D_{v 90 \%}$. Initial value of the dissipation rate of TKE was $\epsilon_{0}=1000 \mathrm{~m}^{2} \mathrm{~s}^{-3}$ at the air injection point, $X / D_{J}=15$. The lines represent the results obtained from the model integrating equation (3.1) and the symbols are the experimental measurements. Experimental Set 3c.

daughter-bubble p.d.f., $f^{*}\left(D^{*}\right)$, and obtain a new number of daughter bubbles until equations (3.4) and (3.5) in conjunction with equation (2.7) for $f^{\prime \prime}\left(D^{\prime \prime}\right)$ are satisfied.

Thus, it is reasonable to expect that our assumption of $m\left(D_{0}\right)=2$ will hold only for small values of $W e_{t}$, and that at large values of $W e_{t}$ the number of daughter bubbles could be greater than two. This increase in the number of daughter bubbles with $W e_{t}$ could explain why we obtained a poorer agreement between the p.d.f. calculated with our model and the experimental measurements for the case of large $W e_{t}$ (see figure 8). Note that, as opposed to the cases where the deformations on the surface of the bubbles were relatively small and the agreement was excellent (figures $5 a, 6 a$ ), now although the agreement is reasonable, there is a noticeable difference between the calculated p.d.f. and the measured ones. To consider a tertiary bubble breakup process, we will further assume, for simplicity, that a bubble is broken into one of diameter $D_{1}$ and two bubbles of equal diameters $D_{2}$, such that

$$
D_{2}=\left[\frac{D_{0}^{3}-D_{1}^{3}}{2}\right]^{1 / 3} \text {. }
$$




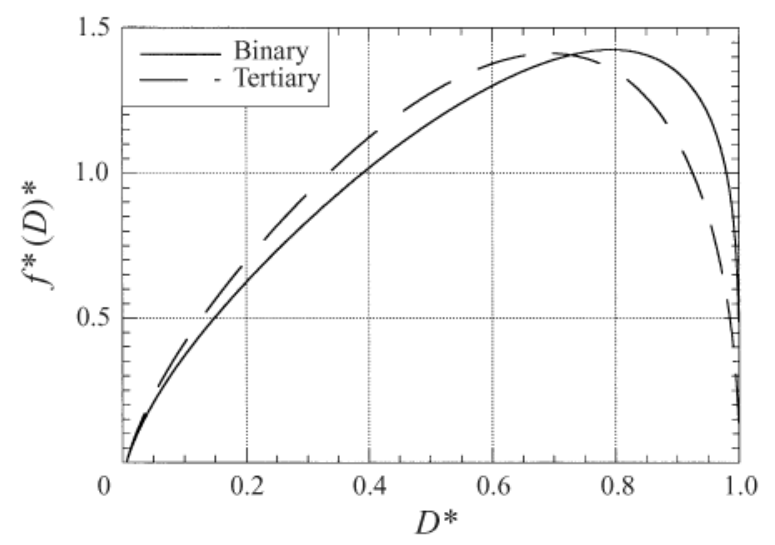

FIGURE 9. Comparison of the daughter-bubble p.d.f. resulting from a binary and a tertiary splitting, $\epsilon=1000 \mathrm{~m}^{2} \mathrm{~s}^{-3}, D_{0}=2 \mathrm{~mm}$.

The daughter-bubble p.d.f. resulting from the breakup of a mother bubble of size $D_{0}$ is then given by

$$
f^{*}\left(D^{*}\right)=\frac{\left[D^{* 2 / 3}-\Lambda^{5 / 3}\right]\left[\left(\frac{1}{2}\left(1-D^{* 3}\right)\right)^{2 / 9}-\Lambda^{5 / 3}\right]^{2}}{\int_{D_{\min }^{*}}^{D_{\max }^{*}}\left[D^{* 2 / 3}-\Lambda^{5 / 3}\right]\left[\left(\frac{1}{2}\left(1-D^{* 3}\right)\right)^{2 / 9}-\Lambda^{5 / 3}\right]^{2} \mathrm{~d}\left(D^{*}\right)},
$$

where $D^{*}=D_{1} / D_{0}, D_{\min } \leqslant D_{1} \leqslant D_{\max }$ and $D_{\max }$ is now given by

$$
D_{\max }=\left[D_{0}^{3}-2\left(\frac{12 \sigma}{\beta \rho D_{0}}\right)^{9 / 2} \epsilon^{-3}\right]^{1 / 3} \text {. }
$$

Equation (3.7) can be used as an extension of our bubble breakup model for cases where the estimated number of bubbles resulting from the breakup is larger than two, although a more elaborate model including all the possible combinations of three bubbles of diameters $D_{1}, D_{2}$ and $D_{3}$ should be considered at larger Weber numbers. The daughter-bubble p.d.f. resulting from equation (3.7) is shown in figure 9 where it is compared to that obtained using a binary breakup assumption for the same flow conditions. Notice that the maximum of the p.d.f. is now shifted to $D^{*} \simeq 0.7$ which corresponds to three bubbles of the same size, and the probability of formation of large bubbles has decreased considerably. Therefore, it is expected to result in a more rapid breakup process than that obtained considering a binary breakup. The downstream evolution of the cumulative volume p.d.f. obtained by integrating equation (3.1) now using the model presented in equation (3.7) is shown, along with the experimental results, in figure 10 for the experimental Set 3c. The agreement between the model and the experimental results is much better, confirming that the number of bubbles created and the shape of the daughter-bubble p.d.f. is strongly dominated by the magnitude of the turbulent stresses.

\subsection{Comparison with other models}

A comparison of the daughter bubble size p.d.f. predicted by our model and those predicted by previously proposed models is shown in figure 11. It is important to emphasize that, as opposed to previously proposed models, the probability density 


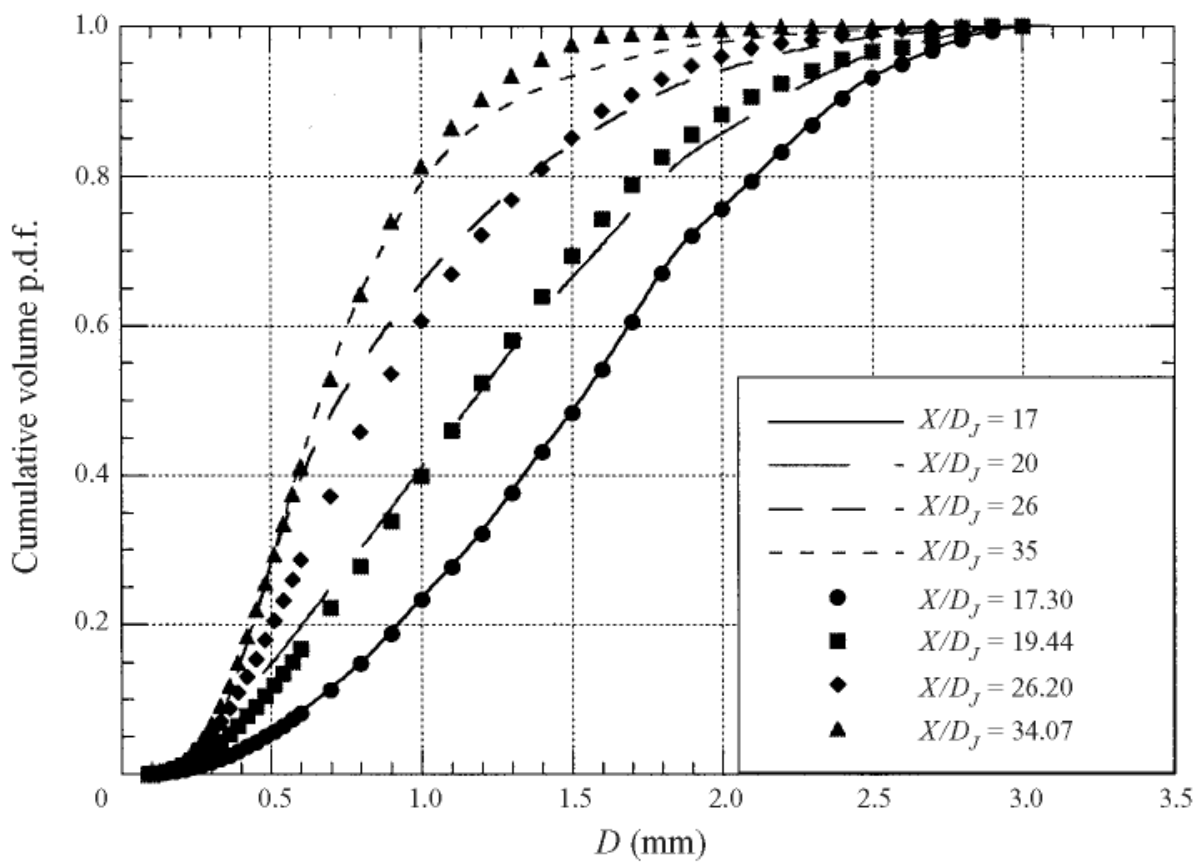

FIGURE 10. Downstream evolution of the cumulative volume probability density function. The lines represent the results obtained using our model assuming a tertiary breakup process model to integrate equation (3.1), and the symbols are the experimental measurements. Experimental Set 3c.

function $f^{*}\left(D^{*}\right)$ given by our model is a function of not only the size of the mother bubble, $D_{0}$, but also of $\epsilon$. To illustrate this point we have plotted $f^{*}\left(D^{*}\right)$ corresponding to three different values of $\epsilon\left(\epsilon=4.5,10\right.$, and $\left.1000 \mathrm{~m}^{2} \mathrm{~s}^{-3}\right)$. As the value of $\epsilon$ increases, $f^{*}\left(D^{*}\right)$ widens out toward smaller sizes, and therefore, the spectrum of bubble sizes resulting from the turbulent breakup of a mother bubble $D_{0}$ is wider, producing smaller bubbles. Note that the model given by Tsouris \& Tavlarides (1994) has a U shape giving a minimum probability for the formation of two daughter bubbles of equal volume $\left(D^{*} \simeq 0.8\right)$, and a maximum probability for the formation of widely different volume pairs.

We have integrated equation (3.1) using Tsouris \& Tavlarides' model in the same manner as we did for our model. The resulting evolution of the cumulative volume p.d.f. is shown in figure 12, where we compare it to the experimental measurements. The discrepancy between the experimental results and the results obtained with this model is very apparent. It is clear that due to the fact that the daughter-bubble p.d.f. is independent of $\epsilon$, this model grossly underpredicts the evolution of the volume p.d.f. The large discrepancy between the measured and calculated evolutions of the $D_{32}$ is due to the fact that this model gives the highest probability to the formation of a very large and very small pair of bubbles, a fact which brings into question the validity of the basic assumptions made in the model.

The downstream evolution of the cumulative volume p.d.f. obtained by integration of equation (3.1), using the daughter-bubble p.d.f. proposed by Konno et al. is shown in figure 13. Although the frozen p.d.f., reached at $X / D_{J}=35$, obtained with this model seems to be in good agreement with the experimental results, the temporal evolution is not very well described. This discrepancy is not surprising since, as shown 


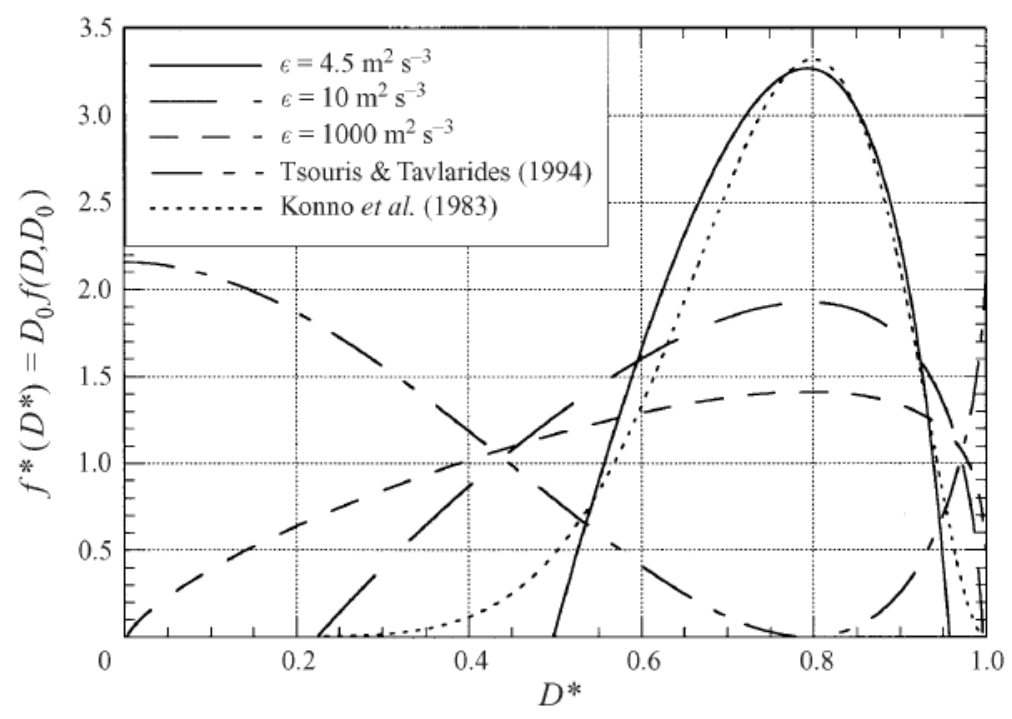

FIGURE 11. Comparison of the daughter bubble size p.d.f. predicted by the present model and previously proposed models by Tsouris \& Tavlarides (1994) and Konno et al. (1983).

in figure 11 , the probability of producing bubbles smaller than $0.3 D_{0}$ after the breakup of a mother bubble of size $D_{0}$ is negligibly small, and independent of the value of both $\epsilon$ and $D_{0}$. Therefore, the size of the daughter bubbles created with this model is always in the range of $0.3 D_{0}<D_{1}<D_{0}$, and it is more likely to form bubbles of similar sizes which translates into a faster evolution of the cumulative volume p.d.f. shown in figure 13. Notice also that in figure 11, the probability of forming small bubbles predicted by our model increases as the value of $\epsilon$ is increased, resulting in a larger amount of small bubbles in the early stages of the breakup process (close to the point of injection) where the value of $\epsilon$ is the largest. As the bubbles are broken, after being injected into the flow by the turbulent stresses of the water jet, they are transported further downstream by the mean motion of the flow to locations of lower value of $\epsilon$. This decrease of $\epsilon$ and $D_{0}$ as the bubbles are convected downstream in the flow produces an evolution of the daughter-bubble p.d.f., increasing the value of the peak and therefore increasing the probability of forming bubbles of equal size. In this evolution, the shape of our daughter-bubble p.d.f. becomes closer to that proposed by Konno et al. This explains why although the calculated cumulative volume p.d.f. obtained using our model is initially different from that obtained using Konno et al.'s model, the final stage of the frozen p.d.f. appears to be well reproduced by both models.

\section{Conclusion}

We have proposed a model for the size p.d.f. of the daughter bubbles produced by the shattering of a mother bubble immersed in a fully developed turbulent water flow. The model is based on the premise that the probability of a given pair forming is proportional to the product of the surplus energy corresponding to the two size scales formed. This model has been shown to be in good agreement with recently obtained experimental data of the transient bubble size distribution. Contrary to previously 

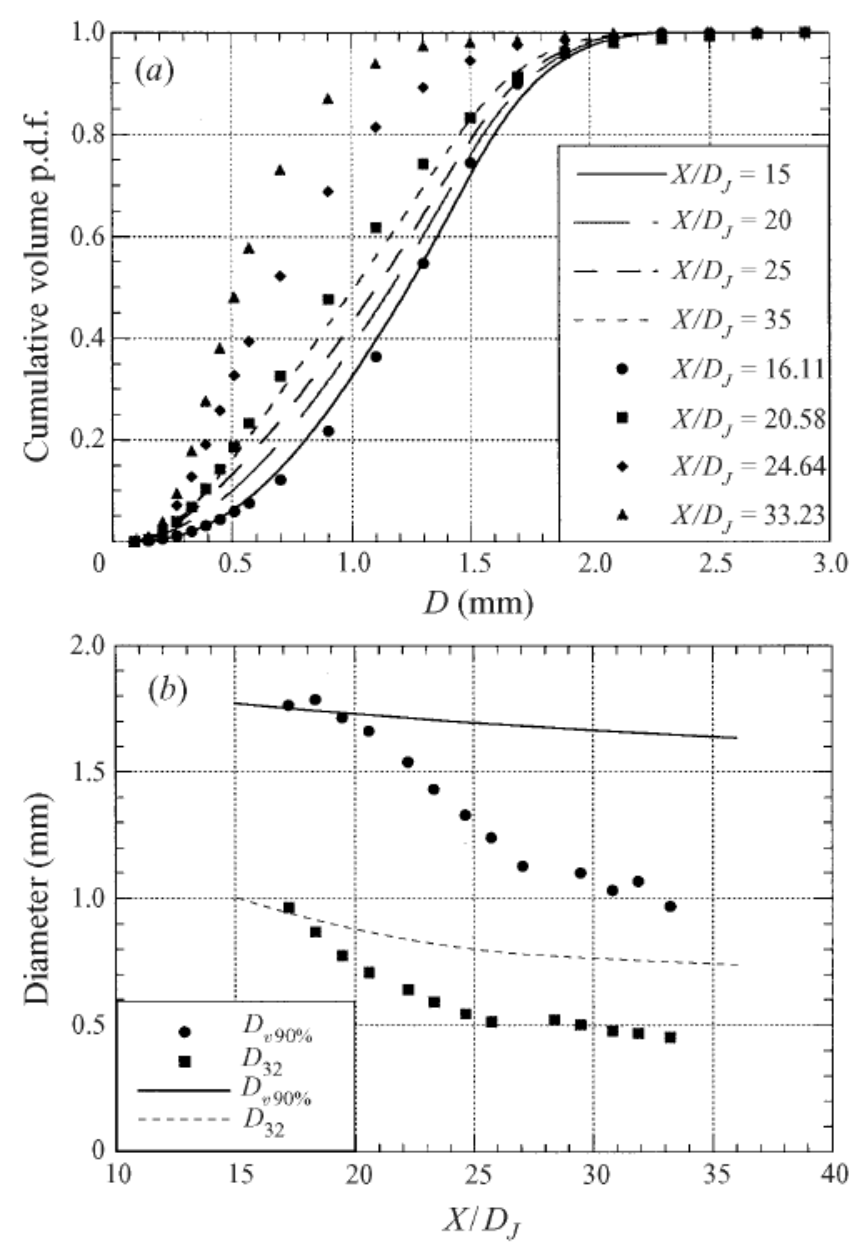

FIGURE 12. (a) Downstream evolution of the cumulative volume probability density function. (b) Downstream evolution of the Sauter mean diameter and $D_{v 90 \%}$. The lines represent the results obtained using Tsouris \& Tavlarides' (1994) model to integrate equation (3.1), and the symbols are the experimental measurements. Experimental Set 2.

existing models, our phenomenological model predicts a dependence of the daughter bubble size p.d.f. on both the bubble size and the value of the dissipation rate of turbulent kinetic energy of the underlying turbulence. The model, although simple and straightforward, predicts, in a consistent manner, the experimental measurements of the transient bubble size p.d.f. performed over a range of bubble sizes and dissipation rates, $\epsilon$. This agreement was found to be better at small and moderate values of the mother bubble's turbulent Weber number in which case the binary breakup assumption was found to be consistent with the experimental observations. At larger turbulent Weber numbers, it was found that the most probable number of bubbles increases, and the assumption of tertiary breakup was found to lead to a better agreement with the experiments.

Support for this work was provided by a grant from the US Office of Naval Research ONR\# N00014-96-1-0213, (Program officer, Dr Edwin P. Rood). The authors are 

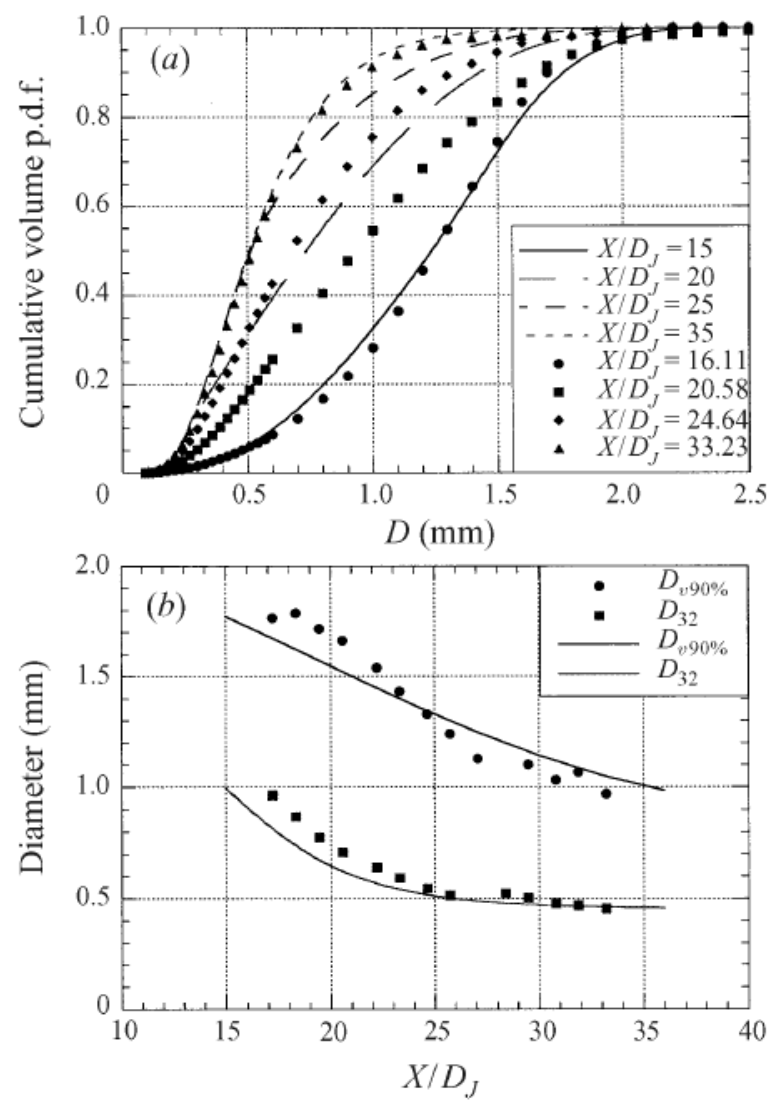

FIGURE 13. (a) Downstream evolution of the cumulative volume probability density function. (b) Downstream evolution of the Sauter mean diameter and $D_{v 90 \%}$. The lines represent the results obtained using Konno et al.'s (1983) model to integrate equation (3.1), and the symbols are the experimental measurements. Experimental Set 2.

grateful for the assistance provided to J. L. M. by the Spanish Ministry of Education while he was on leave from the E.T.S. Ingenieros Aeronáuticos, Universidad Politécnica de Madrid (Spain). The assistance of José Antonio Alfaro, from the Universidad Carlos III de Madrid, is also acknowledged. C. M.-B. would like to thank the support of a Fellowship from the Consejo Asesor the Investigación de la Diputación General de Aragón (Spain). The material presented here has been extracted from the PhD thesis of the first author (C. M.-B.).

This work is dedicated to the memory of Maruja Torralba de Lasheras.

\section{Appendix. On the initial volume distribution of bubbles injected into a turbulent flow}

\section{By M. S. Longuet-Higgins}

Institute for Nonlinear Science, 0402, University of California, San Diego, La Jolla, CA 92093, USA

It may be of interest to compare some of the measurements of bubble distributions described in Part 1 with a much simplier theoretical model proposed earlier by the 

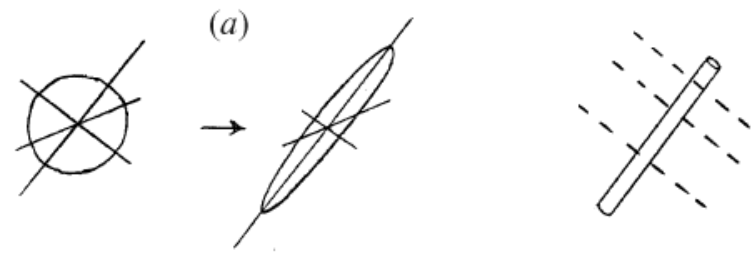

(b)

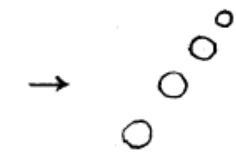

FIGURE 14. Idealized model of the initial bubble breakup.

present author (Longuet-Higgins 1992). The latter can be expected to apply only to the initial distribution of bubble sizes very close to the point of injection, and not to the subsequent stages of development of the bubble distribution. The argument is as follows.

One would expect that any small spherical cavity in a highly turbulent liquid flow would tend to be distorted by the straining of the surrounding flow. In a regime where viscous stresses are negligible, the cavity would tend to be elongated in the direction of the principal rate of strain which has the greatest positive magnitude, as sketched in figure 14 $(a)$. If the form of the cavity is idealized as a cylinder of constant cross-section, it may be assumed to be broken into a small number of pieces by a sequence of $m$ cuts along its length. Figure 14(b) sketches the case $m=3$, for example. If the spacing of the cuts occurs at random, then the volumes of the cuts will be approximated by the one-dimensional 'broken-stick' distribution. What is this?

Let $V_{0}$ be the initial volume of the cavity and $V$ the volume of an individual bubble fragment. Then the p.d.f. of $V / V_{0}$ which we denote by $p\left(V / V_{0}\right)$ is simply

$$
p\left(V / V_{0}\right)=m\left(1-V / V_{0}\right)^{m-1}
$$

as proved for example in Longuet-Higgins (1992). The cumulative distribution of $V / V_{0}$, which we write $P\left(V / V_{0}\right)=\int_{0}^{V / V_{0}} p(\lambda) \mathrm{d}(\lambda)$, is thus given by

$$
P\left(V / V_{0}\right)=1-\left(1-V / V_{0}\right)^{m} .
$$

The quantity shown in figure $9(a)$ of Part 1 is

$$
F(D)=\frac{\mathrm{d} P(V)}{\mathrm{d} D}
$$

where $D$ is the notional diameter of a bubble of volume $D$ that is

$$
V=\frac{\pi}{6} D^{3}, \quad D=(6 V / \pi)^{1 / 3}
$$

If we denote by $D_{0}$ the value of $D$ corresponding to $V_{0}$, then we have

$$
V / V_{0}=\left(D / D_{0}\right)^{3} \text {. }
$$

Writing $V / V_{0}=V^{*}$ and $D / D_{0}=D^{*}$ we find from (A 3) that

$$
D_{0} F(D)=\frac{\mathrm{d} P\left(V^{*}\right)}{\mathrm{d} D^{*}}=\frac{\mathrm{d} V^{*}}{\mathrm{~d} D^{*}} \frac{\mathrm{d} P\left(V^{*}\right)}{\mathrm{d} V^{*}} .
$$

Since

$$
\frac{\mathrm{d} V^{*}}{\mathrm{~d} D^{*}}=3 D^{* 2}
$$

and $\mathrm{d} P\left(V^{*}\right) / \mathrm{d} V^{*}=p\left(V^{*}\right)$ we have altogether

$$
D_{0} F(D)=3 m D^{* 2}\left(1-D^{* 3}\right)^{m-1} \text {. }
$$




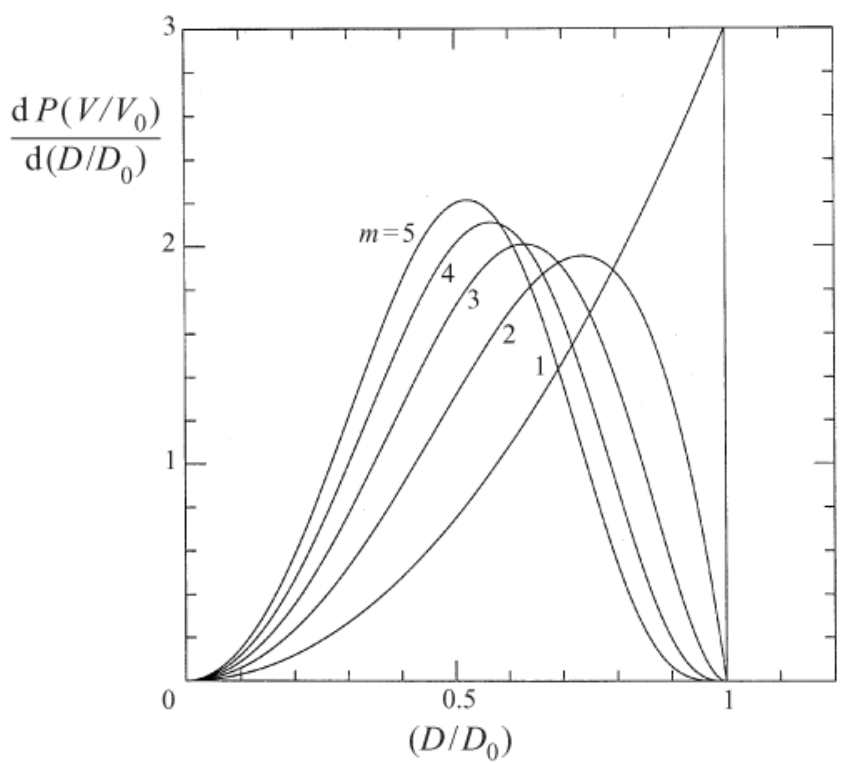

FIGURE 15. The broken-stick volume distribution when $m=1,2, \ldots 5$.

$\begin{array}{cccc}m & D_{m} / D_{0} & D_{0} F\left(D_{m}\right) & D_{m} F\left(D_{m}\right) \\ 1 & 1.0000 & 3.000 & 3.000 \\ 2 & 0.7368 & 1.954 & 1.440 \\ 3 & 0.6300 & 2.009 & 1.266 \\ 4 & 0.5665 & 2.109 & 1.195 \\ 5 & 0.5228 & 2.213 & 1.157\end{array}$

TABle 1. Parameters for the mode of the 'broken-stick' distribution, equation (A 8).

\begin{tabular}{cccccccc}
\hline $\begin{array}{c}\text { Figure } \\
\#\end{array}$ & $\begin{array}{c}D_{a} \\
(\mathrm{~mm})\end{array}$ & $\begin{array}{c}U_{a} \\
\left(\mathrm{~m} \mathrm{~s}^{-1}\right)\end{array}$ & $R_{e}$ & $\begin{array}{c}D_{0} \\
(\mathrm{~mm})\end{array}$ & $\begin{array}{c}D_{m} \\
(\mathrm{~mm})\end{array}$ & $D_{m}^{\circ}$ & $m$ \\
$9(a)$ & 0.39 & 9.84 & 25,000 & 2.55 & 1.7 & 0.68 & 2.58 \\
$12(a)$ & 0.39 & 9.84 & 51,000 & 2.40 & 1.3 & 0.54 & 4.53 \\
\multicolumn{7}{l}{ TABLE } & 2. Parameters of the observed distribution of volume sizes, taken from Part 1.
\end{tabular}

This set of curves is shown in figure 15 for $m=1$ to 5 . For the larger values of $m$ these curves become roughly symmetric in shape. On the left they vary as $D^{* 2}$, and on the right as $\left(1-D^{*}\right)^{m-1}$.

To facilitate a comparison with observation, consider the position of the mode of each distribution. In table 1 we show the mode $D_{m}^{*}$ of the distribution (A 8) which is easily calculated as

$$
D_{m}^{*}=\left(\frac{2}{3 m-1}\right)^{1 / 3} \text {. }
$$

In table 2 are shown some parameters of the observed distribution corresponding to figures $9(a)$ and $12(a)$ of Part $1 . D_{0}$ is the estimated maximum bubble diameter, 

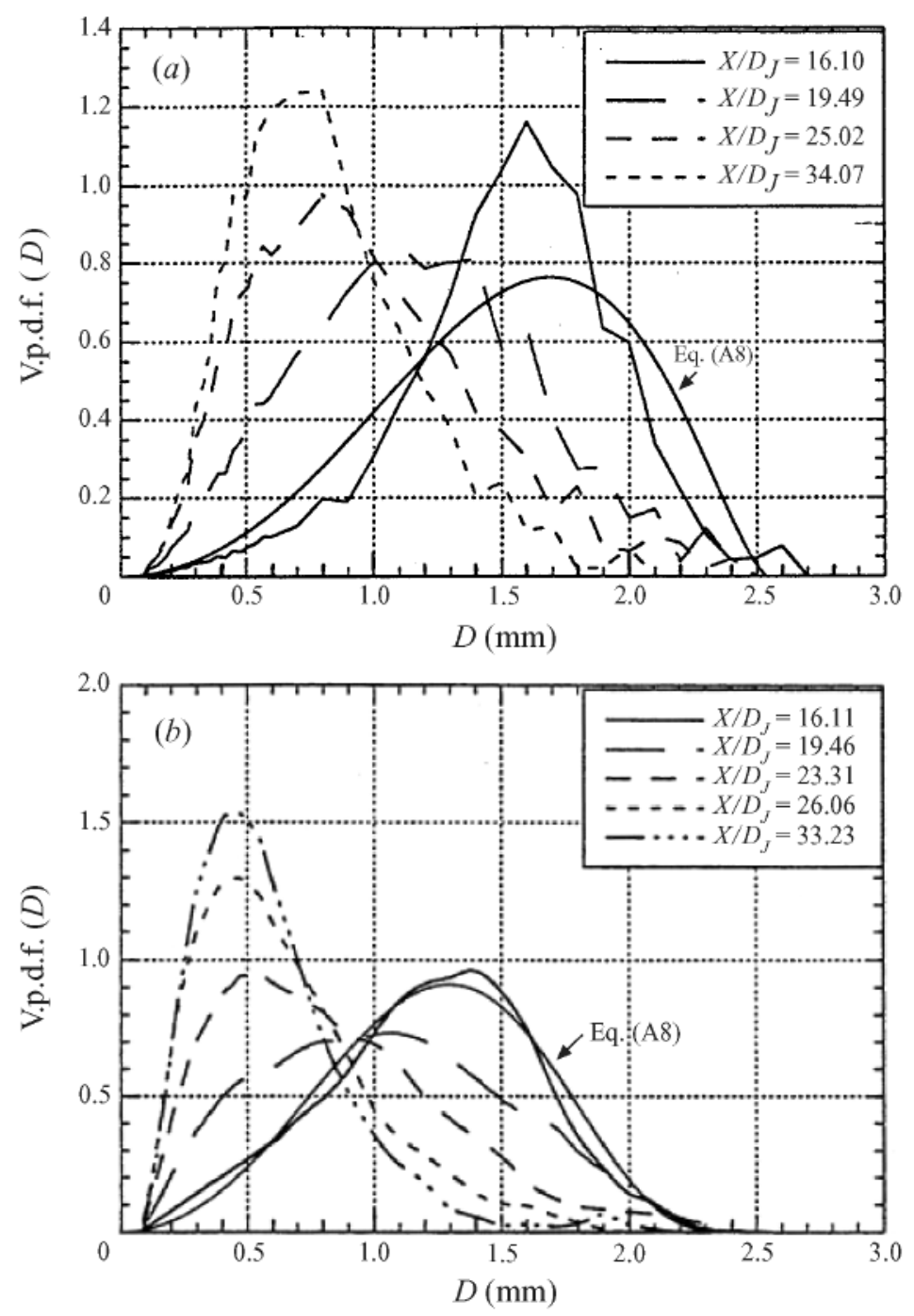

FIGURE 16. The distribution of bubble volumes $(a)$ as shown in figure $9(a)$ of Part 1 , compared with the initial distribution given by equation (A 8), when $D_{0}=2.55$ and $m=2.58$; and $(b)$ as shown in figure 12(a) of Part 1, compared with the initial distribution given by equation (A 8), when $D_{0}=2.4$ and $m=4.53$.

and $D_{m}$ the diameter corresponding to the maximum value of $F(D)$. The next column shows the ratio $D_{m}^{*}=D_{m} / D_{0}$ and the last column shows the value of $m$ calculated from equation (A 9), i.e.

$$
m=\frac{1}{3}\left(1+2 / D_{m}^{* 3}\right)
$$

Non-integral values of $m$ may be taken as corresponding to statistical averages.

Figures 16(a) and 16(b) show a comparison of the observed functions $F(D)$ with the two corresponding theoretical curves. In figure $16(b)$, where the observed curve is the smoothest, the theoretical curve is a fairly close fit to the observations. Note that in figures 7 and $10(a)$ of Part 1 a satisfactory estimate of $D_{0}$ could not be given, and 
in figure $11(a)$ the distribution nearest the point of injection is only for the second 'window', not the first.

At larger distances from the point of injection the peak of the observed volume distribution moves further to the left as does that of the broken-stick distribution for larger values of $m$. However it is not to be expected that the broken-stick distribution will apply, and in fact it does not do so. Note that in the limit as $m \rightarrow \infty, P(V)$ is well approximated by

$$
P(V) \sim 1-\mathrm{e}^{-V / V_{1}}
$$

where $V_{1}=V_{0} / m$, and hence

$$
D_{1} \frac{\mathrm{d} P}{\mathrm{~d} D} \sim 3\left(D / D_{1}\right)^{2} \mathrm{e}^{1\left(D / D_{1}\right)^{3}}
$$

where $D_{1}=D_{0} / \mathrm{m}^{1 / 3}$. On the other hand the limiting distributions shown in Part 1 are generally broader, with longer 'tails' on the right. The peak of the distribution (A 12) occurs when $D / D_{1}=(2 / 3)^{1 / 3}=0.8736$ and so $D \mathrm{~d} P / \mathrm{d} D=2 \mathrm{e}^{-2 / 3}=1.0268$. This is the limit of the values shown in the last column of table 1 . The observed values of $D \mathrm{~d} P / \mathrm{d} D$ lie generally outside this range.

\section{REFERENCES}

Batchelor, G. K. 1956 The theory of Homogeneous Turbulence. Cambridge University Press.

Cohen. R. D. 1991 Shattering of a liquid drop due to impact. Proc. R. Soc. Lond. A 435, 483-503.

Coulaloglou, C. A. \& Tavlarides. L. L. 1977 Description of interaction processes in agitated liquid-liquid dispersions. Chem. Engng Sci. 32, 1289-1297.

FARMER, D. M. \& VAGLE, S. 1988 On the determination of breaking surface wave distribution using ambient sound. J. Geophys. Res. 93, 3591-3600.

Hesketh, R. P., ETchells, A. W. \& Russell, T. W. F. 1991 Bubble breakage in pipeline flow. Chem. Enging Sci. 46, 1-9.

HINZE, J. O. 1955 Fundamentals of the hydrodynamics mechanisms of splitting in dispersion process AIChE J. 1, 289-295.

Kolmogorov, A. N. 1949 On the breakage of drops in a turbulent flow. Dokl. Akad. Naok. SSSR $66,825-828$

KonNo, M., Aoki, M. \& SAITo. S. 1983 Scale-effect on breakup process in liquid-liquid agitated tanks. J. Chem. Engng Japan 16, 312-319.

Konno, M., MatsunaGa, Y., ARaI, K. \& SaITo, S. 1980 Simulation model for breakage process in an agitated tank. J. Chem. Engng Japan 13, 67-73.

Kostoglou, M. \& Karabelas, A. J. 1998 On the attainment of steady state in turbulent pipe flow of dilute dispersions. Chem. Engng Sci. 53, 505-513.

Longuet-Higgins, M. S. 1992 The crushing of air cavities in a liquid. Proc. R. Soc. Lond. A 439, $611-626$.

MARTínez-BAZÁN. C. 1998 Splitting and dispersion of bubbles by turbulence. PhD thesis, University of California, San Diego.

Martínez-BazÁn, C. Montañés, J. L. \& Lasheras, J. C. 1999 On the breakup of an air bubble injected into a fully developed turbulent flow. Part I: Breakup frequency. J. Fluid Mech. 401, $157-182$.

Melville, W. K. 1996 The role of surface-wave breaking in air-sea interaction. Ann. Rev. Fluid Mech. 18, 279-321.

Novikov, E. A. \& Dommermuth, D. G. 1997 Distribution of droplets in a turbulent spray. Phys. Rev. E 56, 5479-5482.

Prince, M. J. \& BLANCH, H. W. 1990 Bubble coalescence and breakup in air-sparged bubble columns. $A I C h E$ J. 36, 1485-1499.

Sathyagal. A. N. \& Ramkrishna. D. 1996 Droplets breakage in stirred dispersions. Breakage functions from experimental drop-size distributions. Chem. Engng Sci. 51, 1377-1391. 
THORpe, S. A. 1982 On the clouds of bubbles formed by breaking wind-waves in deep water, and their role in air-sea gas transfer. Phill. Trans. R. Soc. Lond. A 304, 155-210.

Tsouris, C. \& TAVlarides, L. L. 1994 Breakage and coalescence models for drops in turbulent dispersions. $A I C h E J$. 40, 395-406.

VAlENTAS, K. J. \& Amudson, N. R. 1966 Breakage and coalescence in dispersed phase systems. Engng Chem. Fundam. 5, 533-542.

WiLliams. F.A. 1985 Combustion Theory, 2nd Edn. Addison-Wesley. 